EPJ Web of Conferences 41, 07020 (2013)

DOI: $10.1051 /$ epjconf/20134107020

C Owned by the authors, published by EDP Sciences, 2013

\title{
Mixed Potential Energy Surfaces of the Ultrafast Isomerization of Retinal in Bacteriorhodopsin
}

\author{
Philip J. M. Johnson ${ }^{1}$, Alexei Halpin ${ }^{1}$, Takefumi Morizumi ${ }^{2}$, Valentyn I. Prokhorenko ${ }^{3}$, Oliver P. \\ Ernst $^{2,4}$, and R. J. Dwayne Miller ${ }^{1,3, a}$ \\ ${ }^{1}$ Institute for Optical Sciences and Departments of Chemistry and Physics, University of Toronto, 80 \\ St. George Street, Toronto, Ontario M5S 3H6, Canada, ${ }^{2}$ Department of Biochemistry, University of \\ Toronto, 1 King's College Circle, Toronto, Ontario M5S 1A8, Canada, ${ }^{3}$ Max Planck Research De- \\ partment for Structural Dynamics, Department of Physics, University of Hamburg, Centre for Free \\ Electron Laser Science, DESY, Notkestrasse 85, D-22607 Hamburg, Germany, ${ }^{4}$ Department of Medi- \\ cal Genetics, University of Toronto, 1 King's College Circle, Toronto, Ontario M5S 1A8, Canada
}

\begin{abstract}
We observe, using electronic two-dimensional photon echo spectroscopy, that the cis and trans potential energy surfaces of the ultrafast isomerization of retinal in bacteriorhodopsin are mixed via the hydrogen out of plane (HOOP) mode.
\end{abstract}

\section{Introduction}

The ultrafast isomerization reaction of retinal in bacteriorhodopsin (bR) is a model system for the primary events underlying our sense of vision. To better understand the role quantum dynamics plays in this process, we performed electronic two-dimensional photon echo (2DPE) spectroscopy on bR reconstituted in lipid nanodiscs. These lipid nanodiscs greatly reduce scattering in the sample, resulting in an enhancement of the signal to noise in our measurements by a factor of $\sim 10$ over previous efforts with $\mathrm{bR}$ in native purple membranes [1].

\section{Experimental Methods}

The electronic 2DPE spectrometer used for these measurements has been described in detail previously [2]. A noncollinear optical parametric amplifier (NOPA) with a central wavelength of $545 \mathrm{~nm}$ and a bandwidth supporting $10 \mathrm{fs}$ optical pulses (see Fig. 1) was used to measure the 2DPE spectrum of bR trimers reconstituted in lipid nanodiscs [3], with pulse energies of $\sim 5 \mathrm{~nJ}$ per beam. The sample (OD 0.25 in a $400 \mu \mathrm{m}$ cuvette) was held at room temperature, rotated in the plane normal to the optic axis, and kept under constant illumination of a halogen lamp to keep the sample in the light adapted (all-trans) state. Two-dimensional spectra were collected for waiting times between 0 and 3 ps.

\section{Results and Discussion}

Select electronic 2DPE spectra (the total real signal) are shown in Fig. 2, with waiting times indicated in the lower right corner of each frame. At $T=0 \mathrm{fs}$, where pulse overlap effects are pronounced, we observe a structured 2D spectrum consisting of a $\sim 1000 \mathrm{~cm}^{-1}$ vibrational mode (indicating a HOOP vibration [4]) along the diagonal and negative amplitude signals off the diagonal, which are normally attributable to the instantaneous solvent and glass response. By $10 \mathrm{fs}$, where pulse overlap effects are

\footnotetext{
a e-mail: dwayne.miller@mpsd.cfel.de
} 


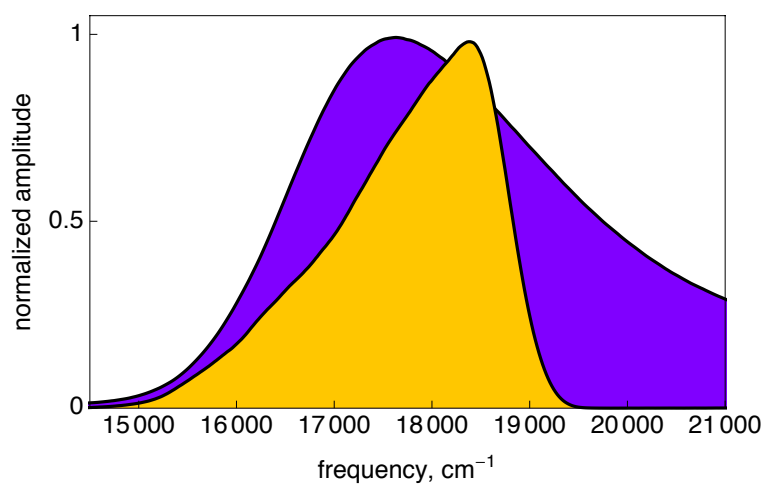

Fig. 1. Absorption profile of bR in lipid nanodiscs with the laser spectrum overlayed, showing the frequency window over which $2 \mathrm{D}$ spectra are collected.

diminished, a pronounced cross peak is observed between the two on-diagonal features, while the large negative amplitude component below the diagonal has all but disappeared. The negative amplitude feature above the diagonal has, however, grown in volume, strongly implying it is not merely a pulse-overlap effect. This component is observed to have its maximal spectral amplitude at the 13-cis absorption maxima, and is fully correlated with the on-diagonal HOOP oscillation. At $25 \mathrm{fs}$, the wave packet on the excited state surface is quickly red-shifting (as was observed in transient-absorption measurements [5]), resulting in a loss of negative amplitude signal and a broad, featureless 2D spectrum.

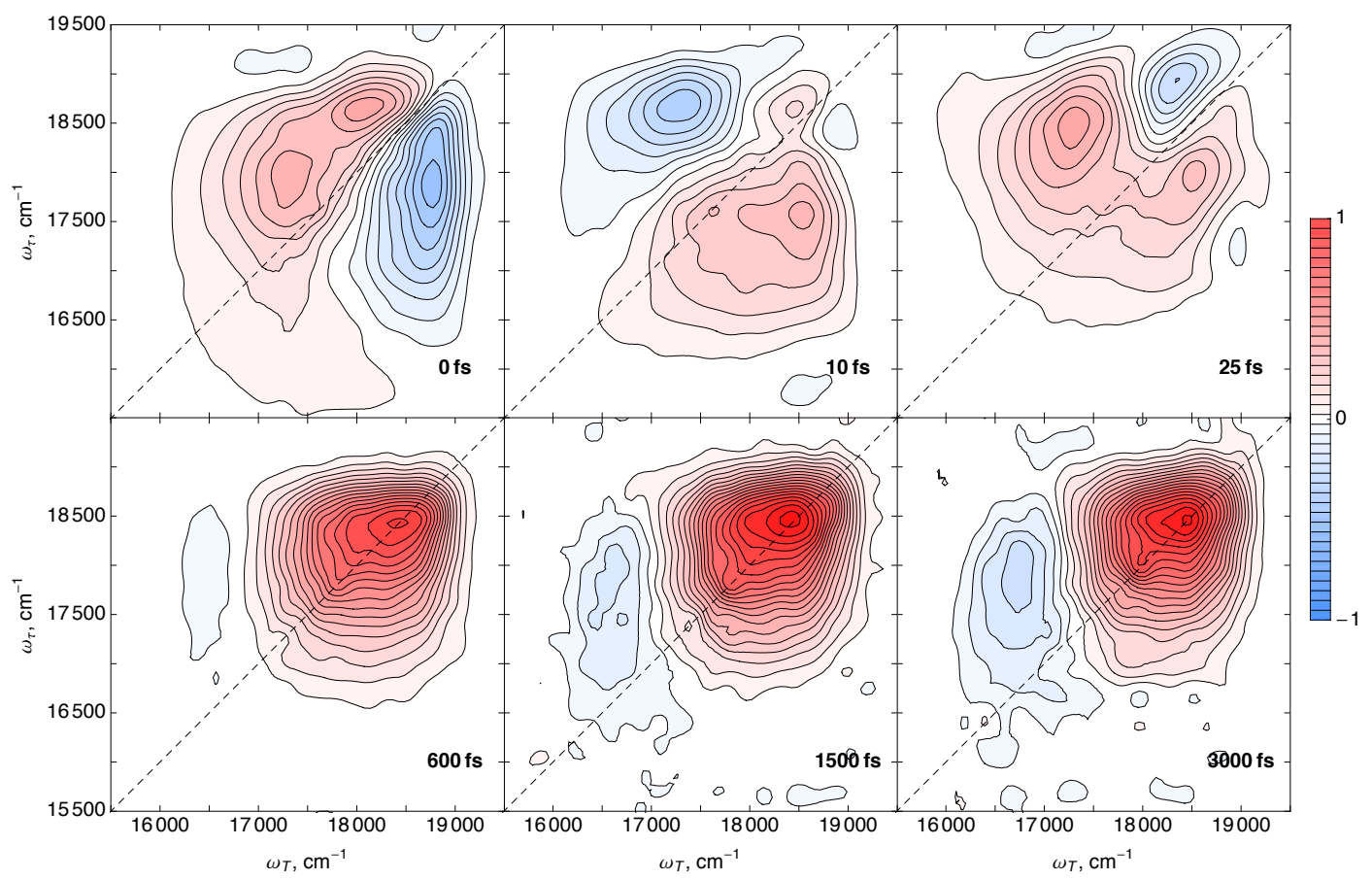

Fig. 2. Time series of the total real component of the 2DPE spectra of the isomerization of retinal in bR. Waiting times are indicated in each frame. At early waiting times (seen most clearly in the $T=10$ fs spectrum) a large negative amplitude feature is observed which is not attributable to pulse overlap effects, and is in spectral concurrence with the known 13-cis absorption spectrum, which appears as a result of excitation at the HOOP feature. 


\section{XVIIIth International Conference on Ultrafast Phenomena}

With the red-shifted wave packet outside of the spectral window set by the NOPA, we observe a strong ground state bleach signal which undergoes spectral diffusion. By a waiting time of $600 \mathrm{fs}$, we see a homogeneously broadened spectrum for the ground state bleach, with an asymmetry due only to the limited spectral bandwidth in the blue. We also observe a negative absorptive feature, now at the differential absorption maximum corresponding to bR's J intermediate, or the 13-cis isomer, as is observed in transient-absorption measurements. As the waiting time continues to increase, we observe a growing in of this $\mathrm{J}$ intermediate, and by $3 \mathrm{ps}$, the $\mathrm{K}$ intermediate has fully formed.

\section{Conclusion}

The strong correlation between the HOOP vibrational mode and a negative amplitude absorptive feature at such early waiting times implies a dynamic mixing of the potential energy surfaces associated with the all-trans and 13-cis retinal isomers at waiting times long before the 13-cis state is populated (by $T=600 \mathrm{fs}$ ). This coupling between states was not observed previously in transient-absorption spectroscopy, but recent theoretical calculations for the isomerization reaction in rhodopsin show that the HOOP mode is the crucial factor in determining the isomerization yield [6,7]. This implies that the HOOP mode is indeed directly coupled to product formation, and is in good agreement with our observation of the characteristic 13-cis state absorption at the onset of the isomerization reaction in bR where the HOOP amplitude is at its largest.

\section{References}

1. V. I. Prokhorenko, A. Halpin, L. S. Brown, and R. J. Dwayne Miller, Ultrafast Phenomena XVII (Oxford University Press, NY, 2011) 580-582.

2. V. I. Prokhorenko, A. Halpin, and R. J. Dwayne Miller, Optics Express 17, (2009) 9764-9779.

3. T. H. Bayburt, Y. V. Grinkova, and S. G. Sligar, Archives of Biochemistry and Biophysics 450, (2006) 215-222.

4. G. R. Loppnow and R. A. Mathies, Biophysical Journal 54, (1988) 9764-9779.

5. R. A. Mathies, C. H. Brito Cruz, W. T. Pollard, and C. V. Shank, Science 240, (1988) 777-779.

6. I. Schapiro, M. N. Ryazantsev, L. M. Frutos, N. Ferré, R. Lindh, and M. Olivucci, Journal of the American Chemical Society 133, (2011) 3354-3364.

7. O. Weingart, P. Altoè, M. Stenta, A. Bottoni, G. Orlandi, and M. Garavelli, Physical Chemistry Chemical Physics 13, (2011) 3645-3648. 